\title{
Impact of COVID-19 on a Tertiary Otolaryngology Practice in Singapore
}

Jian Li Tan, ${ }^{1}$ MBBS (Singapore), MRCS (Glasgow), FAMS (ORL), Ming Yann Lim,${ }^{1}$ MBBS (Melbourne), FAMS (ORL), FRCS ORL (England), Si Ying Chrisanda Lee, ${ }^{1}$ MBBS (Singapore), MRCS (England), Seng Beng Yeo, ${ }^{1}$ MBBS, FRCS (Edin), FAMS (ORL)

\begin{abstract}
The COVID-19 pandemic has had a major impact in healthcare systems across the world, with many hospitals having to come up with protocols and measures to contain the spread of the virus. This affects various specialties' clinical practices in many ways. Since early 2020 in Singapore, the Department of Otorhinolaryngology at Tan Tock Seng Hospital had to rapidly adapt to this pandemic as we provided services to the main healthcare facility combating the virus in our country. We had to design new workflows and also remain flexible in view of the ever-changing situation. There are 6 important domains for an otolaryngology department or any clinical department in general to consider when making adjustments to their practices in an outbreak: (1) clinical work, (2) education, (3) research, (4) safety of patients and staff, (5) morale of medical staff and (6) pandemic frontline work. We hope that the sharing of our experiences and the lessons learnt will be useful for both our local and international colleagues.
\end{abstract}

Ann Acad Med Singap 2020;49:879-901

Keywords: ENT, pandemic, SARS-CoV-2

The Severe Acute Respiratory Syndrome Coronavirus 2 (SARS-CoV-2) that causes Coronavirus disease 2019 (COVID-19) was declared a global pandemic by the World Health Organization (WHO) on 11 March 2020. ${ }^{1}$ Singapore diagnosed its first case of COVID-19 on 23 January 2020 and the first local transmission was reported on 7 February $20200^{2,3}$

In Singapore, the National Centre of Infectious Diseases (NCID) is the designated frontline healthcare facility for treating patients with COVID-19., ${ }^{4,5}$ NCID is physically linked to Tan Tock Seng Hospital (TTSH), which supports the NCID and was also the designated hospital treating severe acute respiratory syndrome (SARS) in 2003. ${ }^{6,7}$ The Department of Otorhinolaryngology at TTSH provides outpatient and inpatient services to TTSH (1,500-bed capacity) and NCID (330-bed capacity); both combine to form the largest inpatient healthcare facility fronting the pandemic in Singapore.

The Department of Otorhinolaryngology had to rapidly adapt to this pandemic by designing and tweaking new workflows as the situation evolved. These are 6 important domains for an otolaryngology department to consider when making adjustments to any outbreak or future pandemic: (1) clinical work, (2) education, (3) research, (4) safety of patients and staff, (5) morale of medical staff and (6) pandemic frontline work.

Clinical work. Appropriate distribution of manpower and resources was required when the pandemic hit, to strike a balance between fighting the pandemic and maintaining our business-as-usual operations.

We initially decreased our new non-urgent outpatient quota by about $50 \%$ for these reasons: (1) reduction of crowding in clinic, (2) manpower deployment to NCID for COVID-19 screening, (3) reduction in elective operating theatres (OTs) due to deployment of anaesthetists to intensive care units (ICUs) treating COVID patients, (4) mandatory 14-day leave of absence for nurses who returned from China after visiting their families over Chinese New Year.

As the situation worsened, we had to commit more manpower to the frontline (NCID), reduce elective

${ }^{1}$ Department of Otorhinolaryngology, Tan Tock Seng Hospital, Singapore

Address for Correspondence: Dr Jian Li Tan, Department of Otorhinolaryngology, Tan Tock Seng Hospital, 11 Jln Tan Tock Seng, Singapore 308433.

Email: Jian_Li_TAN@ttsh.com.sg 
OTs further due to deployment of more anaesthetists to ICUs, and eventually stop accepting non-urgent primary care referrals altogether. Disease Outbreak Response System Condition (DORSCON) is a colour-coded system (green, yellow, orange and red) used to assess the severity and spread of any infectious disease in Singapore. ${ }^{3}$ Singapore raised the DORSCON level to Orange on 7 February, which meant additional measures had to be implemented for large-scale events, daily health checks at workplace, enhanced business continuity capabilities and raised protection for vulnerable groups. ${ }^{3}$

An observation at the start of the outbreak was that the no-show rate for patients was about $30 \%$. This dropped to 10 percent after the government introduced a nationwide abolishment of non-essential services (Fig. 1). The no-show rate would vary depending on the patient's subspecialty problem in a clinic session, as well as the cultural perspectives of the general patient population. This will be a useful factor for consideration in planning clinic manpower in future pandemics, when predicting patient load for manpower allocation purposes.

As far as possible, patients attending clinic appointments were redistributed to other doctors when some otolaryngology colleagues were posted to the frontline. Two 'floating' specialists cover the clinic specially for doctors rostered to the NCID screening centre.

Our allocated elective surgery OT time was significantly reduced as anaesthetists were transferred to augment the ICUs in NCID. As a result, urgent surgery for cancer and airway problems was prioritised.

To ensure the safety of otolaryngology surgeons in OT, different levels of personal protective equipment (PPE) were mandated, depending on the surgical procedure being carried out. ${ }^{8,9,10}$ Table 1 illustrates the various categories of surgery performed by the otolaryngologist and the associated aerosolisation risk, with recommended level of PPE. This table was adopted from another publication by our unit. ${ }^{11}$

Level 1: Surgical mask, eye protection, disposable gloves, cap and gown

Level 2: Fitted N95 mask (National Institute of Occupational Safety and Health-certified), eye protection, disposable gloves, cap and gown

Level 3: Powered Air-Purifying Respirator (eye protection, disposable gloves, cap and gown

Besides the physical components of PPE mentioned above, fit-testing of N95 masks, the correct use and disposal of PPE, as well as meticulous hand hygiene were also crucial. ${ }^{12}$

Other than appropriate PPE for aerosol generating procedures (AGPs), other measures we implemented included minimising the number of personnel in the OT, such as requiring surgeons to be away during intubation and extubation, as these were considered AGPs. ${ }^{8}$ Anaesthetists were also required to use PPE while intubating and extubating.

For COVID-19 patients requiring prolonged intubation, our NCID ICU physicians favoured open tracheostomy to percutaneous tracheostomy for various reasons. ${ }^{13,14}$ Given the ear, nose and throat (ENT) surgeons' expertise in open tracheostomies, we were the designated department to perform this surgery, with special protocols in place. ${ }^{15}$

Education. Our department is heavily involved with postgraduate residency training and undergraduate

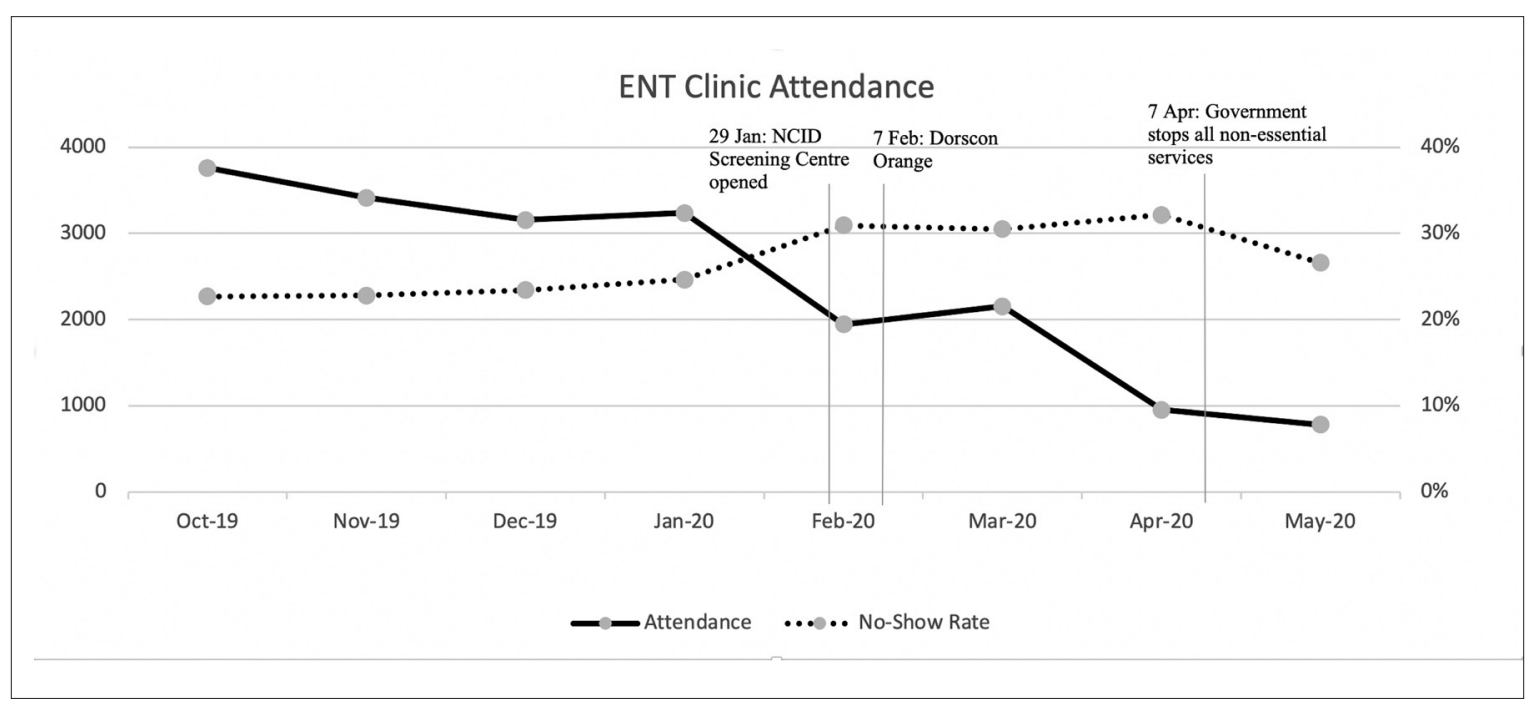

Fig 1. Trajectory of Tan Tock Seng Hospital otolaryngology department's outpatient load 
Table 1. Levels of personal protection equipment to be used in relation to the type of surgery/procedure

\begin{tabular}{lll}
\hline Type of Procedure & General public & COVID-19 Positive/High risk $^{* *}$ \\
\hline Airway procedures & Level 2 & Level 3 \\
\hline Oropharyngeal procedures & Level 2 & Level 3 \\
\hline Otological procedures" & Level 2 & Level 3 \\
\hline Sinonasal procedures & Level 2 & Level 3 \\
\hline Head and neck procedures without breach of aerodigestive tract" & Level 1 & Level 2 \\
\hline "With exceptions & & \\
${ }^{* *}$ High risk according to Ministry of Health suspect case definition & &
\end{tabular}

medical education. For residents, their examinations were postponed for 6 months because of the disruption in training, as well as to avoid the intermingling of residents and examiners during the examinations.

Weekly national resident teaching sessions were converted from attendance at a single physical lecture hall to teaching via teleconferencing. This helped to reduce physical contact and allowed residents to stay in their parent hospital without travelling. The utility and effectiveness of this move is currently being written up for publication by our Otolaryngology Residency Program Director.

For medical students, clinical and bedside teaching was suspended. Tutorials for medical students were conducted via teleconferencing, made possible by information technology support from the hospital and medical schools.

Research. While the COVID-19 outbreak results in reduced clinical work, it also presents new and expanded research opportunities for academia. Our department has written several papers related to this pandemic and we have also engaged the research arm of NCID for other projects.

Safety of patients and staff. During an outbreak, it is very important that patients at a medical facility are not exposed to other patients who may be infected. ${ }^{16}$ In our hospital, all visiting outpatients were screened and their temperature taken at the hospital entrance. Personal details, recent travel overseas or visits to known local COVID-19 hotspots were collected to facilitate contact tracing. The process would be repeated at our clinic entrance and any patient who failed the screening process would be immediately isolated in a predesignated room while awaiting further assessment.

Seating in the otolaryngology clinic outpatient waiting area was rearranged at least $1 \mathrm{~m}$ apart to avoid close proximity between patients, and social distancing was enforced within the clinic. Each patient was only allowed one accompanying relative, who would undergo the same screening process. Our department developed guidelines to determine urgent and non-urgent ENT conditions to help postpone and cut down outpatient appointments, thus restricting people movement.

Otolaryngologists may be at particularly high risk to infection, even when performing previously routine procedures such as flexible nasoendoscopy that can result in viral transmission. ${ }^{17}$ In our clinic, we designated 2 rooms for some procedures previously performed in the clinic consult room. These rooms have High Efficiency Particulate Air filter installed, as well as air exchange and cycling of more than 20 times per hour. Procedures were performed with full PPE and the equipment trolleys and other exposed surfaces would be wiped down with $70 \%$ isopropyl alcohol in between cases. High-risk, non-time-sensitive AGPs were deferred to a later date to conserve valuable PPE resources.

Inpatient ward rounds were restricted to just essential personnel. However, night call teams remained the same to continue the provision of 24-hour emergency services. Surgical face masks were worn by staff during the rounds on non COVID-19 patients. For inpatients with COVID-19, level 2 PPE was worn with specific doffing and donning protocols based on our institution's infection control guidelines. Multidisciplinary meetings such as tumour board and sub-speciality board discussions were converted to either teleconferencing discussions or were held in larger rooms with mandatory wearing of surgical masks and social distancing. This allowed us to continue a high standard of care for patients, even if they had urgent or complex problems during a COVID-19 outbreak.

Morale of medical staff. The morale of staff can be affected during such a trying period, with the mental and emotional burden of potentially getting infected when working regularly with COVID-19 patients. $^{18,19}$ Thankfully, following the public's support for healthcare 
workers during SARS in 2003, Singapore's healthcare workers have received much positive feedback from the public during the COVID-19 outbreak. Many organisations offered discounts on goods and services, as well as donated complimentary food and drinks to our staff. ${ }^{20}$ A notice board was set up in our clinic to display supportive messages from patients and members of the public.

Pandemic frontline work. Since the start of the outbreak, an important and crucial source of medical manpower that allowed for quick ramping up of services and facilities at NCID came from TTSH located close by. The hospital's medical manpower was deployed to the screening centre, inpatient wards and also ICU.

Doctors from the otolaryngology department, as part of the Division of Surgery, contributed as frontline doctors at the NCID screening centre. Working under the Emergency Department consultants, we assessed patients for their presenting symptoms, travel or contact history, as well as physical and imaging findings in order to stratify their risk of COVID-19.

Although participation was voluntary, there was unity and solidarity in contribution, where the head of the department performed the same duties as a junior staff.

Otolaryngology doctors in the screening centre noticed the difficulty many nurses faced in conducting nasopharyngeal swabs for COVID-19 test, partly due to incorrect technique. They quickly collaborated with nursing educators to produce new educational materials such as videos and diagrams to improve the technique and confidence of the staff. Fig. 2 is an extract from the educational videos.

In a pandemic, there will always be many unknowns emerging, resulting in unexpected disruptions to work and lives. As an otolaryngology department providing service to the main healthcare facility in Singapore in

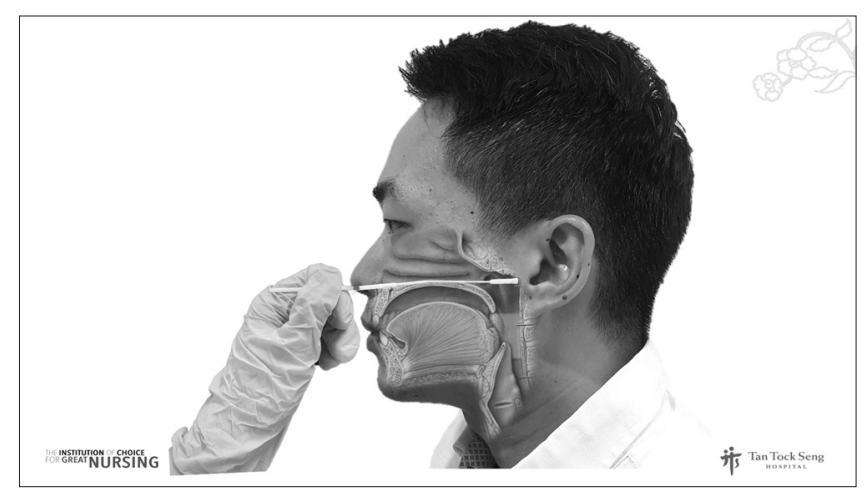

Fig 2. Superimposed diagram to aid in education videos on nasopharyngeal swabs combating the COVID-19 pandemic, we have adjusted our processes to cope with new challenges while still providing continuing care to our ENT patients. Sharing our unique experiences and lessons learnt with colleagues around the world - especially those from the otolaryngology department - will help prepare us all for pandemics in the future.

\section{Acknowledgements}

The authors would like to thank Dr Alex Tham and Adjunct Asst Prof Chong Khai Beng for the figure used for education, as well as Ms Ruth Gao for the clinical data.

\section{REFERENCES}

1. Cucinotta D, Vanelli M. WHO Declares COVID-19 a Pandemic. Acta Biomed. 2020;91:157-60.

2. Hsu LY, Chia PY, Lim JF. The Novel coronavirus (SARS-CoV-2) epidemic. Ann Acad Med Singap 2020;49:1-3.

3. Chen JI, Yap JC, Hsu LY, Teo YY. COVID-19 and Singapore: From Early Response to Circuit Breaker. Ann Acad Med Singap 2020; 49:561-72.

4. Wong JEL, Leo YS, Tan CC. COVID-19 in Singapore-Current Experience: Critical Global Issues That Require Attention and Action. JAMA 2020;323:1243-4.

5. Tan THY, Toh MPHS, Vasoo S, et al. Coronavirus Disease 2019 (COVID-19): The Singapore Experience. A Review of the First Eight Months. Ann Acad Med Singap 2020;49:764-78

6. Seow E. SARS: experience from the emergency department, Tan Tock Seng Hospital, Singapore. Emerg Med J 2003;20:501-4.

7. Tan CK. SARS in Singapore - key lessons from an epidemic Ann Acad Med Singap 2006;35:345-9.

8. Givi B, Schiff BA, Chinn SB, et al. Safety Recommendations for Evaluation and Surgery of the Head and Neck During the COVID-19 Pandemic. JAMA Otolaryngol Head Neck Surg 2020;146:579-84.

9. Workman AD, Welling DB, Carter BS, et al. Endonasal instrumentation and aerosolization risk in the era of COVID-19: simulation, literature review, and proposed mitigation strategies. Int Forum Allergy Rhinol 2020;10:798-805.

10. Workman AD, Jafari A, Welling DB, et al. Airborne Aerosol Generation During Endonasal Procedures in the Era of COVID-19: Risks and Recommendations. Otolaryngol Head Neck Surg 2020;163:465-70.

11. Tan JL, Tay VS, Li H, et al. Otolaryngology Surgery in Time of COVID-19-What PPE to Use When? Ann Acad Med Singap 2020;49:387-92.

12. Mathur P. Hand hygiene: back to the basics of infection control. Indian J Med Res. 2011;134:611-20.

13. Jacob T, Walker A, Mantelakis A, et al. A framework for open tracheostomy in COVID-19 patients. Clin Otolaryngol 2020;45:649-51

14. Portugal LG, Adams DR, Baroody FM, et al. A surgical safety checklist for performing tracheotomy in coronavirus disease 19 patients. Otolaryngol Head Neck Surg 2020;163:42-6.

15. Lim MY, Gan YJ, Tan BH, et al. Practical considerations for the operative team in performing tracheostomy during the COVID-19 pandemic. Singapore Med J 2020. doi: 10.11622/smedj.2020092. 
16. Wang Q, Wang $\mathrm{X}$, Lin $\mathrm{H}$. The role of triage in the prevention and control of COVID-19. Infect Control Hosp Epidemiol 2020;41:772-6.

17. Chan JYK, Wong EWY, Lam W. Practical Aspects of Otolaryngologic Clinical Services During the 2019 Novel Coronavirus Epidemic: An Experience in Hong Kong. JAMA Otolaryngol Head Neck Surg 2020;146:519-20.

18. Zhou Y, Zhou Y, Song Y, et al. Tackling the mental health burden of frontline healthcare staff in the COVID-19 pandemic: China's experiences. Psychol Med 2020;1-2.
19. Chew NWS, Lee GKH, Tan BYQ, et al. A Multinational, Multicentre Study on the Psychological Outcomes and Associated Physical Symptoms Amongst Healthcare Workers During COVID-19 Outbreak. Brain Behav Immun 2020;88:559-65.

20. Goh SSN, Chia MYC. Anxiety and Morale in Front-Line Healthcare Workers During the Coronavirus Disease 2019 (COVID-19) Outbreak at the National Screening Centre in Singapore. Ann Acad Med Singap 2020;49:259-62. 